\title{
Molecular theory of underdamped dielectric relaxation: understanding collective effects in dipolar liquids
}

\author{
B. Bagchi \\ Solid State and Structural Chemistry Unit, Indian Institute of Science, Bangalore 560 012, India
}

and

\author{
A. Chandra \\ Department of Chemistry, University of British Columbia, Vancouver BC, Canada V6T IZI
}

Received 2 November 1992; in final form 11 February 1993

\begin{abstract}
A molecular theory of underdamped dielectric relaxation of a dense dipolar liquid is presented. This theory properly takes into account the collective effects that are present (due to strong intermolecular correlations) in a dipolar liquid. For small rigid molecules, the theory again leads to a three-variable description which, however, is somewhat different from the traditional version. In particular, two of the three parameters are collective in nature and are determined by the orientational pair correlation function. A detailed comparison between the theory and the computer simulation results of Neria and Nitzan is performed and an excellent agreement is obtained without the use of any adjustable or free parameter - the calculation is fully microscopic. The theory can also provide a systematic description of the Poley absorption often observed in dipolar liquids in the high-frequency regime.
\end{abstract}

\section{Introduction}

The dielectric relaxation in a dense dipolar liquid often shows dramatically different behavior at the low- and the high-frequency limits. While the former is often dominated by diffusive motion of the dipolar molecules, the latter is dominated by ultra-fast motions that can be inertial in nature. In many liquids there is a peak in the dielectric absorption spectrum at a relatively high frequency. This absorption, known as Poley absorption, is often attributed to collective excitations, like librations or dipolarons [1-3]. The nature of these excitations is still not clearly understood.

Several somewhat different approaches have been invoked to study the high-frequency dielectric relaxation. In the approach that appears to be pioneered by Rocard [4] many years ago, the dielectric relaxation is described in terms of single-particle rotational motion. For spherical molecules, this approach leads to the following well-known relation:

$$
\frac{\epsilon(\omega)-\epsilon_{\infty}}{\epsilon_{0}-\epsilon_{\infty}}=\frac{1}{\left(1+i \omega \tau_{R}\right)\left(1+i \omega \tau_{J}\right)}
$$

where $\epsilon_{0}$ and $\epsilon_{\infty}$ are respectively the zero- and infinite-frequency limits of the frequency-dependent dielectric function $\epsilon(\omega)$, and $\tau_{R}$ and $\tau_{J}$ are the relaxation times defined by

$$
\begin{aligned}
& \tau_{\mathrm{R}}=\zeta_{\mathrm{R}} / 2 k_{\mathrm{B}} T, \\
& \tau_{J}=I / \zeta_{\mathrm{R}} .
\end{aligned}
$$

In the above equation, $\zeta_{R}$ is the rotational friction on a rotating dipolar molecule, $k_{\mathrm{B}} T$ is Boltzmann's constant times temperature and $I$ is the moment of inertia of a dipolar molecule.

Eq. (1) is known as the Rocard equation. This equation fails to describe the Poley absorption as it predicts a monotonous return to transparency as the frequency is increased. Eq. (1) is a two-parameter theory where the two parameters $\tau_{\mathrm{R}}$ and $\tau_{J}$ are related by the following Hubbard relation [5]: 
$\tau_{\mathrm{R}} \tau_{J}=\frac{I}{2 k_{\mathrm{B}} T}$

The Hubbard relation holds not only for the Langevin-type mechanism, but also for $J$-diffusion type models (see ref. [6] for a discussion of these and related points).

The Rocard equation has been extended in order to account for the Poley absorption. Guillot and Bratos [7] constructed a theory with three independent parameters, $T_{1}=\tau_{\mathrm{R}}, T_{2}=\left(I / 2 k_{\mathrm{B}} T\right)^{1 / 2}$, and $T_{3}=$ $\left(M_{4}-M_{2}\right)^{-1 / 4}$, where $M_{n}$ is the $n$th moment of the infra-red absorption. The second moment $M_{2}$ is equal to $2 k_{\mathrm{B}} T / I$. The fourth moment is given by

$M_{4}=\left(\frac{2 k_{\mathrm{B}} T}{I}\right)^{2}+\frac{2\left\langle N^{2}\right\rangle}{3 I^{2}}$,

where $\left\langle N^{2}\right\rangle$ is the mean-square torque on a molecule. The theory of Guillot and Bratos could provide a semi-quantitative description of the Poley absorption. McConnel [6] has recently provided an elegant analysis of the three-parameter theory where he pointed out that one can recover essentially the Guillot-Bratos result by introducing a finite collision time $\tau_{c}$ in a memory function description of underdamped orientational relaxation.

In a different approach to the study of dielectric relaxation, Madden and Kivelson [8] presented a microscopic study based on the Mori hierarchy [9]. Under appropriate approximations, this theory also reduces to a three-variable theory and the parameters are essentially the same as the ones used in the theory of McConnel [6], except that the approximations are introduced systematically. However, it should be emphasized here that Madden and Kivelson considered dielectric relaxation as the relaxation of the total moment which is different from the single-particle assumption made in all the previous studies. Madden and Kivelson also addressed the question of the existence of collective excitations, such as librations and dipolarons, in the polarization relaxation in dense dipolar liquids and presented certain bounds for the existence of these collective excitations. The main limitation of this otherwise elegant study of Madden and Kivelson is that their theory is not easily amenable to numerical analysis without using experimental parameters and is, therefore, not self-contained.
As in other branches of liquid state physics, computer simulations have played an important role here to unravel the underlying molecular processes involved in dielectric relaxation. The first detailed molecular dynamics simulation of underdamped dielectric relaxation was reported by Pollock and Alder [10]. These authors simulated a Stockmayer liquid. In this liquid, the dipolar molecules interact via a Stockmayer potential (Lennard-Jones + dipole-dipole interaction) which allows the liquid to be in the underdamped limit of orientational relaxation for a rather large range of dipole moment values. The study of Pollock and Alder clearly demonstrated, for the first time, the existence of collective excitations, called dipolarons, in the longitudinal component of the polarization relaxation, but not in the transverse component. However, the Cole--Cole plot clearly indicated the presence of inertial effects in dielectric relaxation in the high-frequency regime. Recently Neria and Nitzan $[11,12]$ have presented a detailed molecular dynamics simulation of the dielectric relaxation in the same Stockmayer liquid. These authors found that for not too strongly polar liquid, the decay of the total moment time correlation function was dominated by a Gaussian component which was followed by an exponential decay at long times. For a strongly polar liquid, the onset of the exponential decay occurs earlier as expected. Another important result is that the rate of Gaussian decay was significantly faster than the free, inertial decay due to temperature alone. This simulation of Neria and Nitzan provides a motivation to develop a molecular theory of dielectric relaxation because a quantitative comparison with theory is now possible. We believe that such a quantitative comparison is presented here for the first time.

Let us now ask the question: what should be the basic features of a first-principles theory of ultra-fast dielectric relaxation? Clearly, only the high-frequency response of the liquid will be involved in this case. Since the dielectric relaxation is related to polarization relaxation and since polarization is related to density relaxation, one would expect a molecular hydrodynamic theory [13] based on the conservation of density and angular momentum to be adequate, provided the short-time response is properly included. Since dielectric relaxation probes the long wavelength orientational relaxation of the system, one 
expects that details of the short-range interactions will not be very important. It is also clear that early decay of the total moment correlation function should be dominated primarily by the static, equilibrium correlations of the liquid. One would then like to have a theory which would pay proper attention to the static orientational correlations present in a dipolar liquid and would include the viscoelastic type responses that would be probed at very short times. It is pertinent to remark at this point that although one is directly investigating a long wavelength property, the shortrange or local correlations can still play an important role through the liquid response functions, as will be explained later.

In this article we present a theoretical study of underdamped dielectric relaxation which uses such a molecular hydrodynamic theory [14] to describe collective orientational relaxation. This approach has been used successfully in recent years to investigate several aspects of polar solvent dynamics [14-16]. In this approach, one uses a time-dependent density functional theory to obtain the collective force on the density fluctuation and a frequency-dependent friction to account for the non-Markovian dissipation of the angular momentum. Secondly, the viscoelastic response of the liquid is included through a frequency-dependent friction. The important thing is that we use a short-time representation for this frequency-dependent friction which is exact in the $t \rightarrow 0$ limit. The novel feature of the present calculation is that for simple, spherical dipolar molecules, it is possible to obtain everything from first principles so that there is no adjustable parameter.

The main results of the present study are the following. The high-frequency dielectric relaxation depends on the following parameters. As before, the inertial time, $\tau_{\mathfrak{l}}$, plays an important role in setting the time scale of relaxation. Secondly, we need the frequency-dependent dielectric friction which is given by the Laplace transform of the torque-torque time correlation function. Lastly, an important role is played by an orientational "caging" parameter, $f^{0}$, which is determined by the long wavelength limit of the orientational correlation function and is related to the static dielectric constant of the medium. In the ultra-short time, the frequency dependent friction is determined by the orientational Einstein frequency, so that our theory becomes a three-variable theory. The orientational Einstein frequency is essentially the mean-squared torque on a tagged dipole and is determined by an integration over the orientational correlation function. The present study also suggests that static (that is, equilibrium) orientational pair correlations play an important role even in the high-frequency dielectric function. Because of the simplicity of the theory and the simplicity of the model potential, a detailed comparison between theoretical predictions and the computer simulation results has been possible. The agreement between theory and simulation is excellent, especially for not too strongly polar dipolar liquids.

The organization of the rest of the paper is as follows. In the next section, we describe the nonMarkovian hydrodynamic theory of dielectric relaxation. In section $3 \mathrm{wc}$ present the detailed numerical work necessary to compare with the simulation of Neria and Nitzan. Section 4 concludes with a brief discussion.

\section{Theory}

The statistical mechanical expressions for frequency $(\omega)$ and wavevector $(\boldsymbol{k})$ dependent dielectric functions have been discussed in several places and we shall present only the necessary equations here. The longitudinal $\left(\epsilon_{\mathrm{L}}(k, \omega)\right)$ and the transverse $\left(\epsilon_{\mathrm{T}}(k, \omega)\right)$ dielectric functions are related to the respective polarizabilities, $\alpha_{\mathrm{L}}(\boldsymbol{k}, \omega)$ and $\alpha_{\mathrm{T}}(\boldsymbol{k}, \omega)$ by the following expressions [14]:

$\epsilon_{\mathrm{L}}(\boldsymbol{k}, \omega)=\left[1-4 \pi \alpha_{\mathrm{L}}(\boldsymbol{k}, \omega)\right]^{-1}$,
$\boldsymbol{\epsilon}_{\mathrm{T}}(\boldsymbol{k}, \omega)=1+4 \pi \alpha_{\mathrm{T}}(k, \omega)$.

The polarizabilities are given by the following linear response relations:

$$
\begin{aligned}
& \alpha_{\mathrm{L}}(\boldsymbol{k}, \omega)=\frac{\beta}{V}\left[C_{P \mathrm{~L}}(\boldsymbol{k}, 0)-\mathrm{i} \omega \tilde{C}_{P \mathrm{~L}}(\boldsymbol{k}, \omega)\right], \\
& \alpha_{\mathrm{T}}(\boldsymbol{k}, \omega)=\frac{\beta}{V}\left[C_{P \mathrm{~T}}(\boldsymbol{k}, \omega)-\mathrm{i} \omega \tilde{C}_{P \mathrm{~T}}(\boldsymbol{k}, \omega)\right],
\end{aligned}
$$

where the correlations functions, $\tilde{C}_{P L}(k, \omega)$ and $\tilde{C}_{P \mathrm{~T}}(k, \omega)$ are defined by the following expressions: 
$\tilde{C}_{P \mathrm{~L}}(\boldsymbol{k}, \omega)=\int_{0}^{\infty} \mathrm{d} t \mathrm{e}^{-\mathrm{i} \omega t}\left\langle P_{\mathrm{L}}(-\boldsymbol{k}, 0) P_{\mathrm{L}}(\boldsymbol{k}, t)\right\rangle$,

$$
\tilde{C}_{P \mathrm{~T}}(k, \omega)=\int_{0}^{\infty} \mathrm{d} t \mathrm{e}^{-\mathrm{i} \omega t}\left\langle P_{\mathrm{T}}(-k, 0) P_{\mathrm{T}}(k, t)\right\rangle
$$

$P_{\mathrm{L}}(k, t)$ and $P_{\mathrm{T}}(k, t)$ are the longitudinal and transverse polarization fluctuations, respectively. The main task, of course, is the evaluation of the polarizability correlation functions. For a system consisting of rigid dipoles, the polarizability is obtained from the position $(r)$ and orientation $(\Omega)$ dependent density,

$$
\boldsymbol{P}(\boldsymbol{r}, t)=\int \mathrm{d} \boldsymbol{\Omega} \boldsymbol{\mu}(\boldsymbol{\Omega}) \rho(\boldsymbol{r}, \boldsymbol{\Omega}, t),
$$

where $\rho(\boldsymbol{r}, \boldsymbol{\Omega}, t)$ is the position ( $\boldsymbol{r}$ ) and orientation $(\Omega)$ dependent number density of the dipolar liquid and $\mu(\Omega)$ is the dipole moment vector with orientation $\boldsymbol{\Omega}$. The wavevector-dependent polarization is obtained by Fourier transforming the space-dependent polarization. Therefore, the calculation of the polarization correlation function is essentially the calculation of the orientational density-density correlation function. The required density-density correlation function can be obtained from a molecular hydrodynamic approach introduced recently by us in the study of the collective orientational relaxation $[14,17,18]$. In this approach, the slow variables are the position- and orientation-dependent number density and the momenta densities. The effects of intermolecular correlations are taken into account via a systematic force term (analogous to the pressure term in the standard Navier-Stokes description) which is given by the density derivative of the free energy functional. The free energy functional is given by the density functional theory [19] which provides a systematic expansion of the free energy in terms of density fluctuation. This approach leads to the following expression for the wavevector and frequency dependent polarization fluctuations:

$$
P_{l}(\boldsymbol{k}, z)=P_{l}(\boldsymbol{k}, t=0)\left(z+\frac{2 f_{l}(k)}{z+\hat{\zeta}_{\mathrm{R}}(z)}+\frac{p q^{2} f_{l}(q)}{z+\hat{\zeta}_{\mathrm{tr}}(z)}\right),
$$

where $i$ stands for either longitudinal (L) or transverse $(T)$ component. The different quantities of eq. (12) are defined as follows:

$f_{\mathrm{L}}(k)=1-\frac{\rho_{0}}{4 \pi} c(110 ; k)$,

$f_{\mathrm{T}}(k)=1+\frac{\rho_{0}}{4 \pi} c(111 ; k)$,

$p=I / m \sigma^{2}, \quad q=k \sigma$,

where $\zeta_{R}(z)$ and $\zeta_{\operatorname{tr}}(z)$ are the frequency-dependent rotational and translational frictions on a dipolar molecule of the liquid, respectively. $c(11 m ; k)$ are the $(11 \mathrm{~m})$ expansion coefficients of the position- and orientation-dependent two-particle direct correlation function of the dipolar liquid of density $\rho_{0}[20]$. In eq. (12), the frequency $z$ is in units of $\tau_{I}$ where $\tau_{I}=\left(I / k_{\mathrm{B}} T\right)^{1 / 2}$.

In order to evaluate the polarization time correlation function, we need the equilibrium, static correlation functions $\left\langle P_{\mathrm{L}}(-k) P_{\mathrm{L}}(k)\right\rangle$ and $\left\langle P_{\mathrm{T}}(-k) P_{\mathrm{T}}(k)\right\rangle$. It is shown elsewhere that these are given by the following expressions [14]:

$$
\begin{aligned}
& \left\langle P_{\mathrm{L}}(-k) P_{\mathrm{L}}(k)\right\rangle=\frac{\rho_{0}}{3}\left(1+\frac{\rho_{0}}{4 \pi} h(110 ; k)\right), \\
& \left\langle P_{\mathrm{T}}(-k) P_{\mathrm{T}}(k)\right\rangle=\frac{\rho_{0}}{3}\left(1-\frac{\rho_{0}}{4 \pi} h(111 ; k)\right),
\end{aligned}
$$

where $h(11 \mathrm{~m} ; k)$ are the $(11 \mathrm{~m})$ expansion coefficients of the total pair correlation function [20]. These coefficients are related to the direct correlation function coefficients $c(11 m ; k)$ via the molecular Ornstein-Zernike relation [20].

For the numerical evaluation of the dielectric function, we, of course, need the values of the orientational correlation function coefficients $c(11 \mathrm{~m} ; k)$ and $h(11 m ; k)$. Fortunatcly, there are now scveral thcoretical methods available for obtaining these functions for dipolar liquids. Among these methods, the most convenient one is the mean spherical approximation (MSA) model [20,21] which was solved by Wertheim [21] to provide a closed-form solution for the orientational pair and direct correlation functions - the solution is especially simple in the Fourier or wavevector $(k)$ space. However, there are two major limitations of MSA. Firstly, it is valid only for dipolar hard spheres whereas we are interested in more 
realistic potentials, especially in this work, in the Stockmayer potential for which computer simulations are available. Secondly, MSA is valid only for weakly polar liquids. In the next section we show how a perturbation scheme can be used to obtain the orientational correlation functions for the Stockmayer liquid using MSA as the reference system. Because we shall use MSA in the numerical calculations, they are limited to weakly polar liquids. However, the theoretical scheme is general.

The important quantity that remains to be specified is the frequency-dependent rotational friction (as we do not need the translational friction to describe the macroscopic (that is, $k=0$ ) dielectric relaxation) and we now discuss the calculation of this quantity. We are interested in the underdamped, inertial decay, and there is a reliable method to calculate the friction coefficient. The method makes use of the following exact relation between the frequency-dependent rotational friction and frequencydependent angular velocity correlation function $C_{\omega}(z)[13]$,

$C_{\omega}(z)=\frac{C_{\omega}(t=0)}{z+\hat{\zeta}_{\mathrm{R}}(z)}$,

where $C_{\omega}(z)$ is defined by $C_{\omega}(z)=\int_{0}^{\infty} \mathrm{d} t$ $\times \exp (-z t) C_{\omega}(t)$ and $C_{\omega}(t)$ is the angular velocity time correlation function. The next important point is that the angular velocity correlation function $C_{\omega}(t)$ admits of the following short-time representation [16]:

$C_{\omega}(t)=C_{\epsilon}(t=0) \exp \left(-\frac{1}{2} \Omega_{\mathrm{R}}^{2} t^{2}\right)$,

which is exact in the $t \rightarrow 0$ limit. Eqs. (15) and (16) together provide the desired value of $\zeta_{R}(z)$. In eq. (16), $\Omega_{R}$ is the orientational Einstein frequency (OEF) of the liquid. We have shown elsewhere that the orientational Einstein frequency of a dipolar liquid is given by the following integral expression $[22,16]$ :

$\Omega_{\mathrm{R}}^{2}=\frac{\rho \mu^{2}}{3 I \pi a^{3}} \int_{0}^{\infty} \mathrm{d} q q j_{1}(q)\left[h_{110}(q)+h_{111}(q)\right]$,

where $j_{1}(q)$ is the spherical Bessel function of the first order and $a$ is the molecular diameter. Eq. (17) is valid when dipolar interaction is the only angle-de- pendent part of the intermolecular potential. The orientational Einstein frequency is essentially the meansquare torque on a molecule, but it is more natural to use the former nomenclature in analogy with the mean square force on a liquid molecule which is called the Einstein frequency [13].

As discussed in the Introduction, all the successful theories of inertial dielectric relaxation seem to involve three parameters. The general form of the present theory is clearly more involved as $f_{L}^{0}$ $\left(=f_{\mathrm{L}}(k=0)\right.$ ) and $\zeta_{\mathrm{R}}(z)$ can hardly be characterized as parameters, as they show complicated dependencies on the structure and the properties of the liquid. However, the theory simplifies considerably in the very (or ultra) short time when the friction coefficient is determined by the Einstein frequency, $\Omega_{R}$. The latter is essentially the mean-square torque that also appears in the theory of Guillot and Bratos.

The present theory provides a rather simple picture of dielectric relaxation in dipolar liquids. The long wavelength polarization relaxation is that of relaxation in an underdamped harmonic oscillator - the force constant of the harmonic well is determined by the static dielectric constant while $\tau_{I}$ gives the relaxation in the absence of the harmonic potential. This is a sensible picture because the friction constant that entered in the previous descriptions should really have no relevance in the underdamped, ultra-fast relaxation as the system can hardly respond to such ultra-fast perturbation. However, the friction plays a very important role at long times.

It is interesting to compare the present theory with that of McConnel [6]. If we ignore the orientational correlations among the dipolar molecules and assume that the torque-torque correlation function decays with a single exponential relaxation time $\tau_{c}$, then the present theory goes over entirely to that of McConnel. However, these approximations may not be correct, for reasons discussed earlier.

In the following we compare the theoretical predictions with the computer simulations because they provide the scope for a detailed and systematic test of the theoretical predictions. A similar comparison with some of the existing experimental results should also be done - we hope to address this issue in the future.

Next we present the results of our numerical calculations. 


\section{Results}

All the numerical calculations presented here are for the Stockmayer liquid which, as mentioned before, is the simplest model for orientational and solvation dynamics because the dipolar interaction is the only angle-dependent intermolecular interaction. We have performed detailed calculations for the parameter values studied by Nitzan and co-workers $[11,12]$. These authors considered two different thermodynamic states of the Stockmayer liquid which we shall refer to as states I and II. The appropriate parameter values for both the states are given in table 1 .

For numerical calculations, we need the values of the dielectric correlation function coefficients $c(110$; $k=0)$ and $c(111 ; k=0)$. These are not readily available for the Stockmayer fluid. However, they can be obtained by mapping the Stockmayer liquid into a system of dipolar hard spheres for which one can use Wertheim's analytical solution of the mean spherical approximation (MSA) model. MSA is known to provide a rather reliable description of the intermolecular correlations for not too strongly dipolar liquids. Therefore, we expect our approach to be fairly accurate for the low polarity state considered by Neria and Nitzan (state I, see table 1). However, this MSA may not be reliable for the second system which is of much higher polarity. For this state, one should perhaps use the reference hypernetted chain (RHNC) model \#1 [23] which, however, requires extensive numerical calculation. In this calculation we use MSA for both the states because of simplicity and transparency that it provides.

We next briefly describe the mapping procedure.

\#1 For a brief discussion on RHNC see ref. [24].

Table 1

The values of the parameters (in reduced units) ") used by Nitzan and co-workers (see refs. [11] and [12]) in their molecular dynamics simulations of Stockmayer liquids. As in the text, the two states simulated are referred to as I and II

\begin{tabular}{lllll}
\hline & $\rho_{\mathbf{L}}^{*}$ & $T^{*}$ & $I^{*}$ & $\mu^{*}$ \\
\hline state I & 0.81 & 1.23 & 0.038 & 1.32 \\
state II & 0.822 & 1.15 & 0.025 & 1.73 \\
\hline
\end{tabular}

a) $\rho_{\mathrm{L} J}^{*}=\rho \sigma_{\mathrm{LJ}}^{3}, T^{*}=k_{\mathrm{B}} T / \epsilon, I^{*}=I / M \sigma^{2}$, and $\mu^{* 2}=\mu^{2} / \epsilon \sigma_{\mathrm{L} J}^{3}$.
The Stockmayer liquid is characterized by three dimensionless parameters defined by

$\rho^{*} \equiv \rho \sigma_{\mathrm{LJ}}^{3}, \quad T^{*}=\frac{k_{\mathrm{B}} T}{\epsilon}, \quad \mu^{*}=\left(\frac{\mu^{2}}{\epsilon \sigma_{\mathrm{LJ}}^{3}}\right)^{1 / 2}$,

where $\mu$ is the magnitude of the dipole moment of a molecule, $\epsilon$ and $\sigma_{\mathrm{L}}$ are the usual Lennard-Jones parameters for energy and diameter. On the other hand, a system of dipolar hard spheres is characterized by only two dimensionless quantities, the reduced dipole moment $\mu_{\mathrm{HS}}^{*}$ and the reduced density $\rho^{*}$ which are defined by

$\mu_{\mathrm{HS}}^{*} \equiv\left(\frac{\mu^{2}}{k_{\mathrm{B}} T \sigma_{\mathrm{HS}}^{3}}\right)^{1 / 2}, \rho^{*}=\rho \sigma_{\mathrm{HS}}^{3}$.

$\sigma_{\text {HS }}$ is the diameter of a hard sphere. We use the Verlet-Weis scheme [25] of implementing the Weeks-Chandler-Andersen (WCA) perturbation theory [26] to find the density and temperature dependent effective hard sphere diameter $\sigma_{\mathrm{HS}}$ which maps the Lennard-Jones part of the Stockmayer liquid into the hard-sphere liquid. We find $\mu^{*}=1.17$ and $\rho_{\text {HS }}^{*}=0.83$ for the first system (state I of NN) and $\mu^{*}=1.6$ and $\rho_{\mathrm{HS}}^{*}=0.84$ for the second state. It is straightforward to use MSA to obtain the direct correlation function coefficients. The procedure and the necessary equations have been described in many places $[20,21,14]$.

In fig. 1 we present the total moment-moment correlation function $C_{M}(t)$ for the state $I$ of the Stockmayer fluid considered by Neria and Nitzan. This less polar system shows inertial, Gaussian time dependence for a long time. In this figure we also show the simulation results. The agreement between theory and computer simulation is excellent. In fig. 2 we present the same results for the more polar system the state II of Neria and Nitzan. The more polar system goes over to the exponential decay in a much shorter time. In this case, the agreement between computer simulation and theory is not satisfactory. This lack of agreement can be due to several reasons. Firstly, MSA is not reliable at large polarities. Secondly, the dielectric friction may be rather large for this system and the short time expression for the velocity correlation given by eq. (16) may not be reliable. This deserves further study.

In fig. 3, we show the transverse moment-moment 


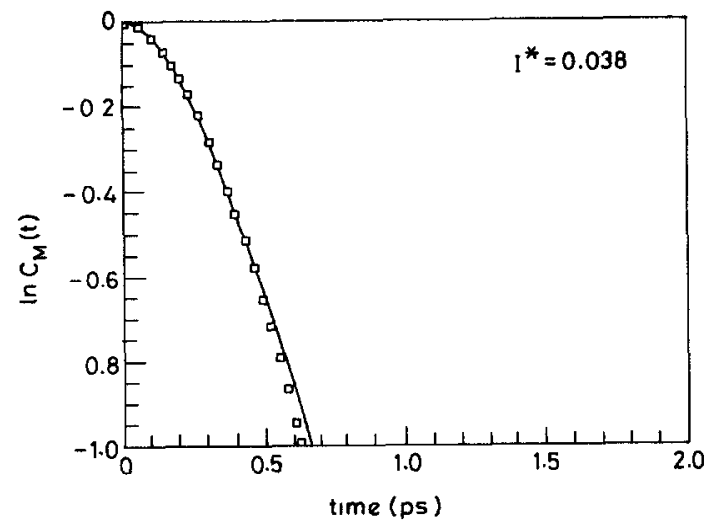

Fig. 1. The total moment time correlation function, $C_{M}(t)$ is plotted against time for the state $I$ of the Stockmayer liquid simulated in ref. [12]. The parameter values for the state I are given in table 1 . The theoretical results are given by the solid line while the simulation results by squares. The details are discussed in the text.

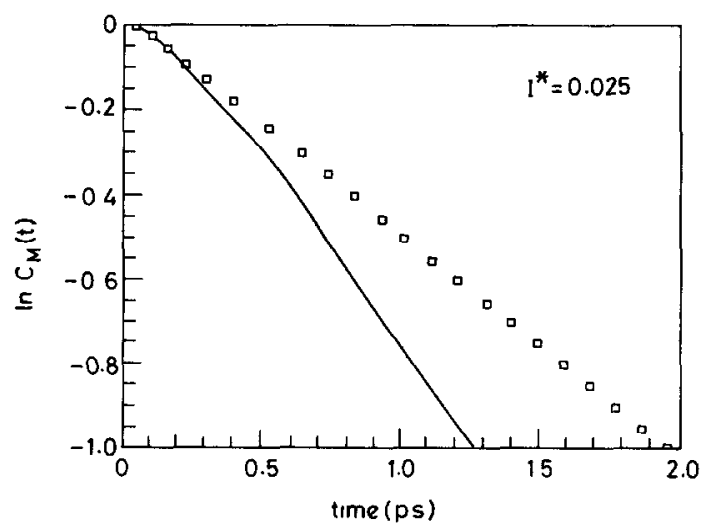

Fig. 2. The total moment time correlation function, $C_{M}(t)$ is plotted against time for the state II of the Stockmayer liquid simulated in ref. [12]. The parameter values for the state I are given in table 1 . The theoretical results are given by the solid line while the simulation results by squares. The details are discussed in the tèxt.

time correlation functions for both the two states and in fig. 4 we show the same but for the longitudinal component. The two are strongly different. While the decay of the transverse component is similar to that of the total moment correlation function, the longitudinal parts show pronounced oscillations. What is more remarkable is that although the transverse relaxations are vastly different for the two states, the longitudinal component shows remarkable similarity

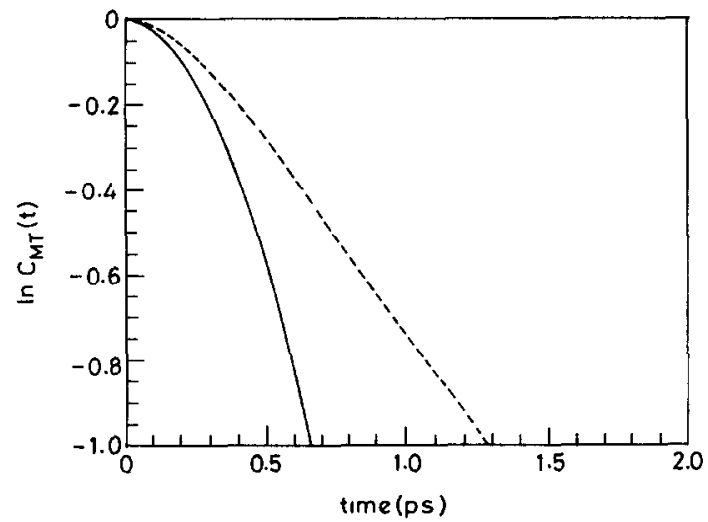

Fig. 3. The transverse moment time correlation function, $C_{M T}(t)$ is plotted against time for both the states I and II of the Stockmayer liquid simulated in ref. [12]. The parameter values for both the states are given in table 1 . The details are discussed in the text.

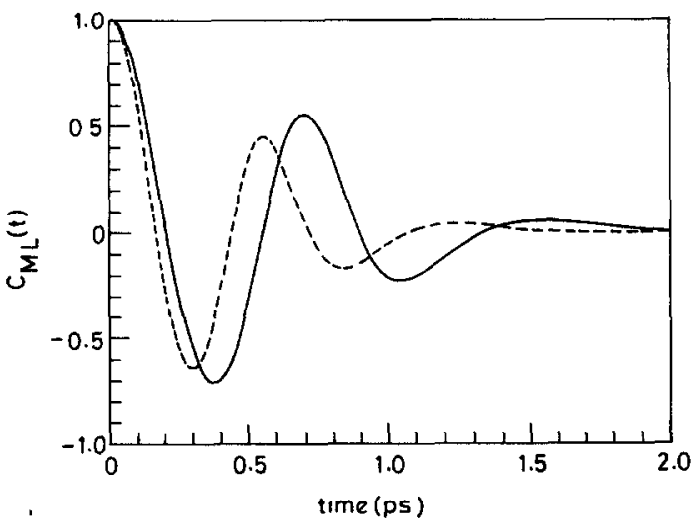

Fig. 4. The longitudinal moment time correlation function, $C_{M T}(t)$ is plotted against time for both the states I and II of the Stockmayer liquid simulated in ref. [12]. The parameter values for both the states are given in table 1 . The details are discussed in the text.

in the relaxation behavior. This might explain the very similar time dependencies in the solvation dynamics of an ion observed by Neria and Nitzan in their computer simulations, although the dielectric relaxation behavior of the two systems was strongly different.

The oscillatory decay of the longitudinal moment correlation function suggests that it may be appropriate to consider this as a collective excitation. Such an excitation is absent for the transverse part. This is in agreement with previous studies $[8,10,18]$.

We now turn to the discussion of the Poley absorption. As shown in fig. 3, the initial decay of the trans- 
verse polarization component also decays with a Gaussian time dependence

$$
C_{P \mathrm{~T}}(k=0, t)=C_{P \mathrm{~T}}(k=0, t=0) \exp \left[-\left(t / \tau_{\mathrm{TG}}\right)^{2}\right] \text {. }
$$

This can be used to estimate the high-frequency dielectric absorption given by the imaginary part of the dielectric function which is now given by the following expression,

$\frac{\epsilon^{\prime \prime}(\omega)}{\epsilon_{0}-1}=\frac{2}{\tau_{\mathrm{TG}}^{2}} \int_{0}^{\infty} \mathrm{d} t(\sin \omega t) t \exp \left[-\left(t / \tau_{\mathrm{TG}}\right)^{2}\right]$.

Eq. (21) has a maximum at a frequency which is, approximately, inversely proportional to $\tau_{\mathrm{TG}}$. The maximum at such a high frequency is attributed to the Poley absorption. Note that $\tau_{\mathrm{TG}}$ is similar in value to $\tau_{I}$.

\section{Conclusion}

In this article we have presented a molecular hydrodynamic theory of ultra-fast underdamped dielectric relaxation in a dense dipolar liquid. The merit of this description is that for a liquid of small rigid molecules it is possible to offer detailed predictions without using any adjustable parameter. For the model dipolar liquid, we find excellent agreement between theory and computer simulation results, especially for a system of low polarity.

The structure of the general theory is similar to the theory of Madden and Kivelson [8], except that the present molecular hydrodynamic approach is simpler, transparent and amenable to numerical calculations. This is primarily due to the use of the density functional theory where the two-particle direct correlation function, rather than the radial distribution function naturally enters the description. Also, the density expansion of the free energy gives rise to a convolution integral form which enables us to exploit the translational isotropy of the system by working in the Fourier space.

The theory predicts that in the short-time relaxation of an underdamped liquid, the macroscopic friction (or the viscosity) of the liquid does not play any significant role. Rather, it is the static intermolecular orientational correlations that determine the rate of the relaxation.

The use of the short-time approximation (eq. (16)) somewhat limits the applicability of the present approach. As we have already pointed out, the main reason for its use is that it allows the calculations to remain fully microscopic and self-contained. This is because we can calculate the orientational Einstein frequency exactly for the Stockmayer liquid. Although this short-time approximation works extremely well for the less polar system, it does not provide a satisfactory description of the more polar liquid. It is certainly an interesting problem to consider a more appropriate description of the frequency-dependent friction. Another interesting problem is to consider real liquids where short-range attractive interactions can be important. This can give rise to librational modes at low temperatures. In such cases, the description of the friction is going to be more complicated.

\section{Acknowledgement}

We thank Professor A. Nitzan for sending preprints of his work prior to publication. This work was supported in part by a grant from the Department of Science and Technology, Government of India.

\section{Referencesc}

[1] R. Lobo, J.E. Robinson and S. Rodriguez, J. Chem. Phys. 59 (1973) 5922.

[2] G. Ascarelli, Chem. Phys. Letters 39 (1976) 23.

[3] W.G. Rothschild, Dynamics of molecular liquids (WileyInterscience, New York, 1984).

[4] Y. Rocard, J. Phys. Radium 4 (1933) 247.

[5] P.S. Hubbard, Phys. Rev. 131 (1963) 1155.

[6] J. McConnel, J. Mol. Liq. 48 (1991) 99.

[7] B. Guillot and S. Bratos, Phys. Rev. A 16 (1977) 424.

[8] P. Madden and D. Kivelson, Advan. Chem. Phys. 56 (1984) 467.

[9] H. Mori, Progr. Theoret. Phys. 33 (1965) 423; 34 (1965) 399.

[10] E.L. Pollock and B.J. Alder, Phys. Rev. Letters 46 (1981) 950.

[11] N. Neria and A. Nitzan, J. Chem. Phys. 96 (1992) 5433.

[12] N. Neria, A. Nitzan, R.N. Barnett and U. Landman, preprint, to be published in the Proceedings of UPS, 1991. 
[13] P. Boon and S. Yip, Molecular hydrodynamics (McGrawHill, New York, 1980).

[14] B. Bagchi and A. Chandra, Advan. Chem. Phys. 80 (1991) 1.

[15] B. Bagchi, Annu. Rev. Phys. Chem. 40 (1989) 115.

[16] B. Bagchi and A. Chandra, J. Chem. Phys. 97 (1992) 5126.

[17] A. Chandra and B. Bagchi, J. Chem. Phys. 91 (1989) 1829.

[18] B. Bagchi and A. Chandra, Phys. Rev. Letters 64 (1990) 455.

[19] J.L. Lebowitz and J.K. Percus, J. Math. Phys. 4 (1963) 116.
[20] C.G. Gray and K.E. Gubbins, Theory of simple liquids, Vol. 1 (Oxford Univ. Press, Oxford, 1984).

[21] M.S. Wertheim, J. Chem. Phys. 55 (1971) 4291.

[22] B. Bagchi, unpublished work.

[23] P.H. Fries and G.N. Patey, J. Chem. Phys. 82 (1985) 429.

[24] J.P. Harsen and I.F. McDonald, Theory of simple liquids (Academic Press, New York, 1986).

[25] L. Verlet and J.J. Weis, Phys. Rev, A 5 (1972) 939.

[26] J.D. Weeks, D. Chandler and H.C. Andersen, J. Chem. Phys. 54 (1971) 5327. 